and $3,39 \%$ were found to have a perinatal/antenatal brain injury etiology. Of the 906 patients who had records for the seizure history, 39\% were diagnosed with epilepsy and $93.77 \%$ of these patients had clinical seizures and an electroencephalography-determined seizure. The rate of global growth retardation was determined as $88.6 \%$ in the patients with developmental evaluation abnormal by DENVER II developmental screening test. When DENVER II findings of the patients with growth retardation were evaluated in terms of area, it was found that $91.1 \%$ of the patients had language, $82.7 \%$ had personal and social, $89.3 \%$ had gross motor and $87.1 \%$ had thin motor field regression. The patients with WISC-R were found to have a mild intellectual disability rate of $51.1 \%(\mathrm{n}=24)$, a moderate mental disability rate of $44.7 \%(n=21)$ and a severe mental deficiency rate of $4.3 \%(\mathrm{n}=2)$ determined. $29.72 \%$ of patients who underwent cranial MRI showed abnormal findings. Cerebral anomaly was found in $94.3 \%$ of patients with abnormal cranial MRI findings, $16.7 \%$ had brain stem dysplasia, $11.6 \%$ had cerebellar anomalies, $1.4 \%$ had pituitary gland hypoplasia and $0.2 \%$ had meningoencephalocele were detected. Therefore, microcephalic patients should be closely monitored in terms of epilepsy, mental insufficiency and global growth retardation, and counseling should be provided to their families. In addition, it was found that cranial MRI was important for the clarification of some etiologies.

\section{P495 IS NARCOLEPSY INCIDENCE INCREASING OR SYMPTOMS JUST BETTER RECOGNISED?}

${ }^{1}$ Jennifer Hayden*, ${ }^{2}$ Lisa Dann, ${ }^{2}$ Nicolas M Allen, ${ }^{3}$ Bryan Lynch. ${ }^{1}$ Dept. of Paediatrics, National University of Ireland Galway, and Galway University Hospita, Galway, Ireland; ${ }^{2}$ Galway University HospitalDept. of Paediatrics, National University of Ireland Galway, and Galway University Hospital (GUH), Galway, Ireland; ${ }^{3}$ Galway University HospitalTemple St. Children's University HospitalTemple St. Children's University Hospital Temple St. Children's University Hospital, Dublin , Ireland

10.1136/archdischild-2019-epa.831

Background Narcolepsy is a rare and under-recognised disease characterised by excessive daytime sleepiness, disturbed nocturnal sleep, hallucinations, and cataplexy, with a major impact on quality of life. Diagnosis can be challenging. We present a series of recent children presenting to a district general hospital over a 2.5 year period with chronic symptoms ultimately diagnosed with narcolepsy.

Patients Patient 1: Female(12y) GP referral with 2-year history of increased sleepiness and falling asleep during meals. Later developed cataplexy-'when I laugh, I go weak suddenly'. Swine-flu vaccine 6 years previously.

Patient 2: Female(8y) GP referral with 18-month history of excessive day-time sleepiness and vivid nightmares. Narcolepsy was considered when seen in clinic initially but significant academic decline subsequently prompted formal diagnosis (9 months after referral). Laugh induced catatonia (eyes roll, tongue protrusion and lower limb weakness). Pandemrix H1N1 vaccine 3 years previously. Sleep study noted abnormal sleep latency and sleep onset REM periods. Improvement with methylphenidate.

Patient 3: Male(10y) emergency department (ED) presentation with a 2-month history of excessive day time sleepiness and increased appetite. Falling asleep while standing occasionally. No cataplexy. Sleep study abnormal (2.3 minutes sleep latency and SOREM periods in $3 / 4$ naps). Treatment with sodium oxybate.

Patient 4: Female(5y) ED presentation with 2-month history of daily episodes of eye rolling and sudden collapse, weeks following MMR. Initial diagnosis and treatment for epilepsy. Video review reveals clinical diagnosis of cataplexy; focused history revealed excessive daytime sleepiness. Treatment with stimulants (methylphenidate) improved sleep symptoms; considering sodium-oxybate for cataplexy.

Patient 5: Male(15y) GP referral with 3 year history of excessive day-time sleepiness, struggling to keep up academically. Upon laughing his mouth droops open with tongue protrusion (cataplexy). No swine-flu vaccine. Treatment pending investigations.

Patients 1-4 had undetectable CSF hypocretin levels (except patient 2; failed LP) and positivity for the HLA-DQB1*0602 association in keeping with narcolepsy. Patient 5 is awaiting specific investigations. Sleep studies are pending for 3 patients. Secondary causes of narcolepsy were out-ruled.

Discussion Delay in narcolepsy diagnosis is associated with worse outcomes. While narcolepsy is thought to be under recognised, these 5 patients were suspected upon referral to paediatrics and management instigated quickly; however focused history and increased awareness is required. Our series and review of a national narcolepsy clinic suggests increased incidence and early detection in children. A national narcolepsy service (currently Temple-St-Hospital, Dublin) facilitates management, as symptoms and co-morbidities can be debilitating and life-long. Vaccine association remains controversial.

\section{P496 X- LINKED INFANTILE SPINAL MUSCULAR ATROPHY (SMAX2) CAUSED BY NOVEL C.1681G>A SUBSTITUTION IN THE UBA1 GENE, EXPANDING THE PHENOTYPE}

Niamh Shaughnessy*, Eva B Forman, Declan O’ Rourke, Sally Ann Lynch, Lynch Bryan. Temple Street Children's University Hospital , Dublin, Ireland

10.1136/archdischild-2019-epa.832

Aims We report the case of a male infant who presented following a normal pregnancy with typical symptoms of X-linked infantile spinal muscular atrophy including hypotonia, weakness, areflexia and respiratory insufficiency, however contractures were absent. X-linked infantile spinal muscular atrophy (SMAX2), OMIM 301830, is a rare, severe form of spinal muscular atrophy, caused by variants in the Ubiquitin like modifier-activating enzyme 1 (UBA1) gene1. Clinical features reported to date include marked hypotonia, areflexia, arthrogryposis, contractures, myopathic facies, tongue fibrillations and cryptorchidism2. To our knowledge, this is the first reported case of SMAX2 to present without contractures.

Methods This infant was born at $40+4$ weeks following a normal pregnancy to non-consanguineous Irish parents. Intermittent stridor was noted following delivery, laryngomalacia was diagnosed following review by ENT. At 8 weeks old there was an acute event at home with colour change and poor feeding for 2 days. On presentation to ED, there was marked increased work of breathing, weakness, absent deep tendon reflexes, hypotonia, myopathic facies and weak cry. Eyes were bright and there was no ophthalmoplegia or contractures. A significant family history of neuromuscular disease on the patient's maternal side was noted, with several male relatives all dying before the age of six months. Creatine Kinase was 